\title{
Immunization in familial amyloidotic polyneuropathy: counteracting deposition by immunization with a Y78F TTR mutant
}

\author{
Hisayasu Terazaki ${ }^{1,2,3}$, Yukio Ando ${ }^{4}$, Rui Fernandes ${ }^{1}$, Ken-ichi Yamamura ${ }^{5}$, \\ Shuichiro Maeda ${ }^{6}$ and Maria J Saraiva ${ }^{1,7}$
}

\begin{abstract}
${ }^{1}$ Molecular Neurobiology, Institute for Molecular and Cell Biology, University of Porto, Porto, Portugal; ${ }^{2}$ Department of Gastroenterology, Institute of Molecular Embryology and Genetics, Kumamoto University, School of Medicine, Kumamoto, Japan; ${ }^{3}$ Department of Hepatology, Institute of Molecular Embryology and Genetics, Kumamoto University, School of Medicine, Kumamoto, Japan; ${ }^{4}$ Department of Diagnostic Medicine, Institute of Molecular Embryology and Genetics, Kumamoto University, School of Medicine, Kumamoto, Japan; ${ }^{5}$ Division of Developmental Genetics, Institute of Molecular Embryology and Genetics, Kumamoto University, School of Medicine, Kumamoto, Japan; ${ }^{6}$ Department of Biochemistry, Yamanashi University Faculty of Medicine, Yamanashi, Japan and ${ }^{7}$ ICBAS, University of Porto, Porto, Portugal
\end{abstract}

\begin{abstract}
The mechanism of amyloid formation in familial amyloidotic polyneuropathy (FAP), a hereditary disorder associated with mutant transthyretin (TTR), is still unknown. It is generally believed that altered conformations exposing cryptic regions are intermediary steps in this mechanism. A TTR mutant- $\mathrm{Y78F}$ (transthyretin mutant with phenylalanine replacing tyrosine at position 78)-designed to destabilize the native structure has been shown to expose a cryptic epitope recognized by a monoclonal antibody that reacts only with highly amyloidogenic mutants presenting the amyloid fold or with amyloid fibrils. To test whether TTR deposition in FAP can be counteracted by antibodies for cryptic epitopes, we immunized with TTR Y78F, transgenic mice carrying the most common FAP-associated TTR mutant-V30M (transthyretin mutant with methionine replacing valine at position 30) - at selected ages that present normally with either nonfibrillar or TTR amyloid deposition. Compared to age-matched control nonimmunized mice, Y78F-immunized mice had a significant reduction in TTR deposition usually found in this strain, in particular in stomach and intestine; by contrast, animals immunized with V30M did not show differences in deposition in comparison with nonimmunized mice. Immunohistochemical analyses of tissues revealed that immunization with $\mathrm{Y78F}$ lead to infiltration by lymphocytes and macrophages at common deposition sites, but not in tissues such as liver, choroid plexus, and Langerhans islets, in which TTR is produced. These results suggest that $\mathrm{Y78F}$ induced production of an antibody that reacts specifically with deposits and leads to an immune response effective in removing/ preventing TTR deposition. Therefore, TTR immunization with selected TTR mutants has potential application in immune therapy for FAP.
\end{abstract}

Laboratory Investigation (2006) 86, 23-31. doi:10.1038/labinvest.3700365; published online 7 November 2005

Keywords: Alzheimer disease; amyloid; familial amyloidotic polyneuropathy; immunization; transthyretin

Various types of amyloidoses have in common an abnormal folding of soluble precursor proteins, which results in formation of extracellular amyloid deposits. ${ }^{1}$ Examples of disorders in which this process occurs are Alzheimer's disease, ${ }^{2}$ priontransmissible spongiform encephalopathies, ${ }^{3}$ and familial amyloidotic polyneuropathy (FAP). ${ }^{4}$

Correspondence: Professor MJ Saraiva, PhD, Instituto de Biologia Molecular e Celular, Molecular Neurobiology, Rua do Campo Alegre 823, Porto 4150, Portugal.

E-mail: mjsaraiv@ibmc.up.pt

Received 3 June 2005; revised and accepted 30 September 2005; published online 7 November 2005
FAP associated with amyloidogenic transthyretin (ATTR) is characterized by amyloid deposition in peripheral nerves, visceral organs, autonomic nervous system, gastrointestinal tract, and ocular tissues. ${ }^{5}$ The disease duration is about 10 years after onset $^{6}$ unless liver transplantation is performed during the early stage. ${ }^{7,8}$ By the end of 2000, more than 500 patients worldwide with FAP had undergone this surgery, with about $80 \%$ of them surviving. ${ }^{8}$ However, there are several problems with this therapy: (1) it is expensive; (2) patients with transplants must continue lifelong administration of immune suppressants after the surgery; (3) both 
carriers of the ATTR gene who do not show clinical symptoms and patients in the advanced stage of FAP cannot undergo liver transplantation; (4) patients with FAP-V30M TTR have been reported to develop vitreous amyloid deposits after liver transplantation, as the result of variant TTR production by the retina. $^{9,10}$ A new strategy for therapy is therefore needed.

The strategies put forward so far as potentially therapeutic for FAP follow for most aspects directions generally taken for other amyloidoses, such as inhibiting aggregation and/or disrupting TTR amyloid by selective molecules. ${ }^{11-13}$ The application of immune therapies currently investigated in Alzheimer and Prion diseases ${ }^{14-16}$ have not to date been considered in FAP.

The mechanism of TTR amyloid formation is still unknown. It is believed that altered conformations exposing cryptic regions are intermediary steps in this mechanism. ${ }^{17}$

A TTR mutant-Y78F (transthyretin mutant with phenylalanine replacing tyrosine at position 78)designed to destabilize the native structure has been shown to expose a cryptic epitope recognized by a monoclonal antibody that reacts only with highly amyloidogenic mutants presenting the amyloid fold or with amyloid fibrils ${ }^{18}$ We believe that Y78F adopts an intermediate structure in the fibrillogenesis pathway and therefore reasoned that the cryptic epitopes displayed by this mutant would trigger tissue-specific immunological reactions as observed in immunization protocols with aggregated a- $\beta$ peptide leading to resolution of deposits both in humans $^{19}$ and in the PDAPP (amyloid $\beta$ precursor protein mini-gene driven by a platelet-derived growth factor promoter) mouse. ${ }^{14}$

We report here results obtained by immunization with TTR Y78F of a transgenic (Tg) mice model for FAP, carrying the most common associated TTR mutant-V30M (transthyretin mutant with methionine replacing valine at position 30)—at selected ages that normally present with either nonfibrillar or TTR amyloid deposition..$^{20,21}$

\section{Materials and methods}

\section{Animals}

Mice used in this study included TTR-null mice ${ }^{22}$ and Tg mice carrying the human TTR V30M gene backcrossed to the TTR-null background. ${ }^{20}$ Animals were kept and used strictly in accordance with National and European Union guidelines for the care and handling of laboratory animals.

\section{Antigens}

Recombinant human wild-type and mutant TTR (V30M and Y78F) were produced in an Escherichia coli system and purified as previously described. ${ }^{18}$ Briefly, after growing the bacteria, the protein was isolated and purified by preparative gel electrophoresis after ion exchange chromatography. Protein concentration was determined using the Lowry method.

\section{Immunization Protocol}

(1) TTR-null mice of 6 months after birth were immunized with human V30M $(n=3)$ or human Y78F $(n=3)$; (2) V30M Tg mice of 6 months after birth were immunized with human V30M $(n=5)$ or Y78F $(n=9)$ or PBS alone $(n=6)$; (3) V30M Tg mice of 12 or 18 months after birth were immunized with phosphate buffer saline (PBS) alone $(n=5$, and 6 , respectively) or human Y78F ( $n=9$ for both ages).

TTR V30M or TTR Y78F were dissolved in PBS at a concentration of $2 \mathrm{mg} / \mathrm{ml}$, emulsified 1:1 (v/v) with complete Freund's adjuvant (Sigma, St Louis, MO, USA) and $100 \mu \mathrm{l}$ of the mixture was injected subcutaneously, followed by a boost dose in incomplete Freund's adjuvant (Sigma, St Louis, MO, USA) 2 weeks later and monthly thereafter. The fifth immunization consisted of V30M or Y78F in PBS alone. At 4 months after vaccination, mice were anesthetized with sodium pentobarbital $(150 \mathrm{mg} / \mathrm{kg}$, intraperitoneally), blood collected, tissues perfused with PBS buffer, excised, fixed in $4 \%$ paraformaldehyde and embedded in paraffin.

\section{Antibody Detection by Immunoassay}

Anti-TTR antibodies were detected by an indirect ELISA protocol. Microtiter plates (NUNC, Roskilde, Denmark) were coated overnight at $4^{\circ} \mathrm{C}$ with rabbit polyclonal antibody anti-human TTR (DAKO, Glostrup, Denmark) at a concentration of $5 \mu \mathrm{g} / \mathrm{ml}$ and blocked with 5\% skimmed milk diluted in PBS. The plates were then incubated with several TTR preparations (wild type, V30M, or Y78F) for $1 \mathrm{~h}$ at $37^{\circ} \mathrm{C}$; after three washes in PBS with $2 \%$ Tween 20, sera from different mice diluted 100 times in PBS were added. Bound antibodies were detected with horseradish peroxidase-labeled antimouse Ig G antibody (Pierce, Rockford, IL, USA; dilution 1:500). Plates were developed using $5 \mathrm{mM}$ 2,2'-azinobis(3-ethylbenzthiazoline-6-sulfonic acid) (ABTS) (Sigma, St Louis, MO, USA) in $50 \mathrm{mM}$ phosphate citrate buffer, $\mathrm{pH}$ 5.2, and optical densities read at $411 \mathrm{~nm}$ on a microplate reader (Bio-Tek, Winooski, VT, USA).

\section{Tissue Analyses}

\section{Congo red (CR) staining}

The presence of amyloid in tissue sections was investigated after staining with CR and observation under polarized light. ${ }^{23}$ 


\section{Immunohistochemical staining}

For immunohistochemistry, $5 \mu$ m-thick sections were deparaffinated in xylol and dehydrated in a descendent alcohol series. Endogenous peroxidase activity was inhibited with $3 \%$ hydrogen peroxide/ $100 \%$ methanol and sections were blocked in blocking solution ( $4 \%$ bovine serum and $1 \%$ bovine serum albumin (BSA) in PBS). Primary antibodies were diluted in blocking solution and incubated overnight at $4{ }^{\circ} \mathrm{C}$. Antibodies used were polyclonal anti-human TTR antibody (DAKO, Glostrup, Denmark, 1:200 dilution), rat antimouse CD45R/B220 and anti-CD5 monoclonal antibodies (BD Pharmigen Europe, 1:50 dilution), and rat anti-CD11b/CD18 (Mac-1) monoclonal antibody (Chemicon, Temecula, CA, USA, 1:100 dilution).

The presence of Ig $\mathrm{G}$ in tissues was investigated by immunohistochemistry using horseradish peroxidase-labeled anti-mouse pan Ig $\mathrm{G}$ antibody (Pierce, Rockford, IL, USA, dilution 1:500).

\section{Tissue amyloid plaque immunoreactivity}

Ig $\mathrm{G}$ from immunized $\mathrm{Tg}$ mice was purified by Protein G Sepharose (Pharmacia) following the instructions of the supplier and tested for reactivity in human pancreas paraffin sections from FAP patients and control individuals. On parallel sections, Ig $G$ preabsorbed with excess TTR $(100 \mu \mathrm{g}$ of recombinant TTR/ $\mu \mathrm{g}$ of antibody) was used.

Antigen visualization was performed with the biotin-extravidin-peroxidase kit (Sigma, St Louis, MO, USA), using 3-amino-9-ethyl carbazole (Sigma, St Louis, MO, USA) or diaminobenzidine as substrates.

The Video finder light (Olympus, Tokyo, Japan) linked to an Olympus microscope (type BX50) through a CCD video camera served for quantification image analysis. We used $\times 200$ magnification for quantification. Semiquantitative analysis of immunohistochemical images was performed with the Universal Imaging System (NIH), which performs automated particle analysis in a measured area, that is, the area occupied by pixels corresponding to the immunohistochemical substrate colour is counted and normalized relative to the total area. Each slide used in semiquantitative immunohistochemistry was analyzed in 15 different selected areas. Before quantification of each slide, the baseline threshold was corrected using negative control slides. Results shown represent \% occupied area \pm s.d.

\section{Statistical Analyses}

Mann-Whitney nonparametric analysis via Microsoft Excel was used, with $P<0.02$ considered as statistically significant.

\section{Results}

\section{Antibody Reactivity}

Sera from immunized TTR-null and V30M Tg mice were assessed by indirect ELISA for their reactivities towards Y78F, V30M, and wild-type TTRs. Sera from TTR-null mice immunized with Y78F or V30M presented significantly higher reactivity towards the TTR antigens than sera from V30M Tg mice immunized with the same preparations, which relates to the absence of transgene-expressed human TTR antigen in the null strain (as shown in Figure 1a).

A difference in signal towards the mutant TTRs was observed among V30M Tg mice: while antibodies from V30M Tg mice immunized with V30M reacted similarly with V30M and Y78F, those resulting from immunization with $Y 78 \mathrm{~F}$ had a higher signal for the Y78F protein than antibodies from V30M Tg mice immunized with V30M, possibly due to the existence of unique antigenic determinants in Y78F.

Antibodies from V30M Tg mice immunized with Y78F were then tested for their ability to recognize TTR in human tissues, either in soluble or fibrillar forms; Figure 1b-e). Figure $1 \mathrm{~b}$ shows a clear staining of TTR amyloid deposits of an FAP pancreas by Ig $\mathrm{G}$ isolated from an V30M Tg mice immunized with Y78F, which overlapped staining with rabbit anti-TTR (for the WT form) in an adjacent section (Figure 1c); a positive reaction was also obtained for soluble TTR produced by pancreas from a normal individual (Figure 1d); specificity of the reaction was demonstrated by absorption of the antibody with soluble TTR, which abolished tissue reactivity (Figure 1e).

\section{Quantitative Assessment of TTR Deposition in Immunized Vs NonImmunized Mice}

Nonfibrillar TTR deposition has been observed in the V30M Tg mice used in this study, particularly in the GI tract with $90 \%$ of animals at 3 months presenting TTR deposits in the stomach. Deposition in the form of amyloid is rarely observed before 12 months, whereas between 13 and 18 months, approximately $50 \%$ of the animals have CR-positive material extending to intestine as a major local of amyloid deposition. ${ }^{21}$

We used histological analyses to compare the effects of immunization with V30M vs Y78F of transgenic animals which started the immunization protocol at the age of 6 months after birth $(n=5$ and 9 , respectively) before deposition of TTR fibrils in tissues; all mice were killed at 10 months after birth and quantitative immunohistochemical measurements were used to determine the amount of amyloid and nonfibrillar TTR deposition.

In all animals and organs examined (esophagus, stomach, intestine, kidney, lung, liver, spleen, heart, 

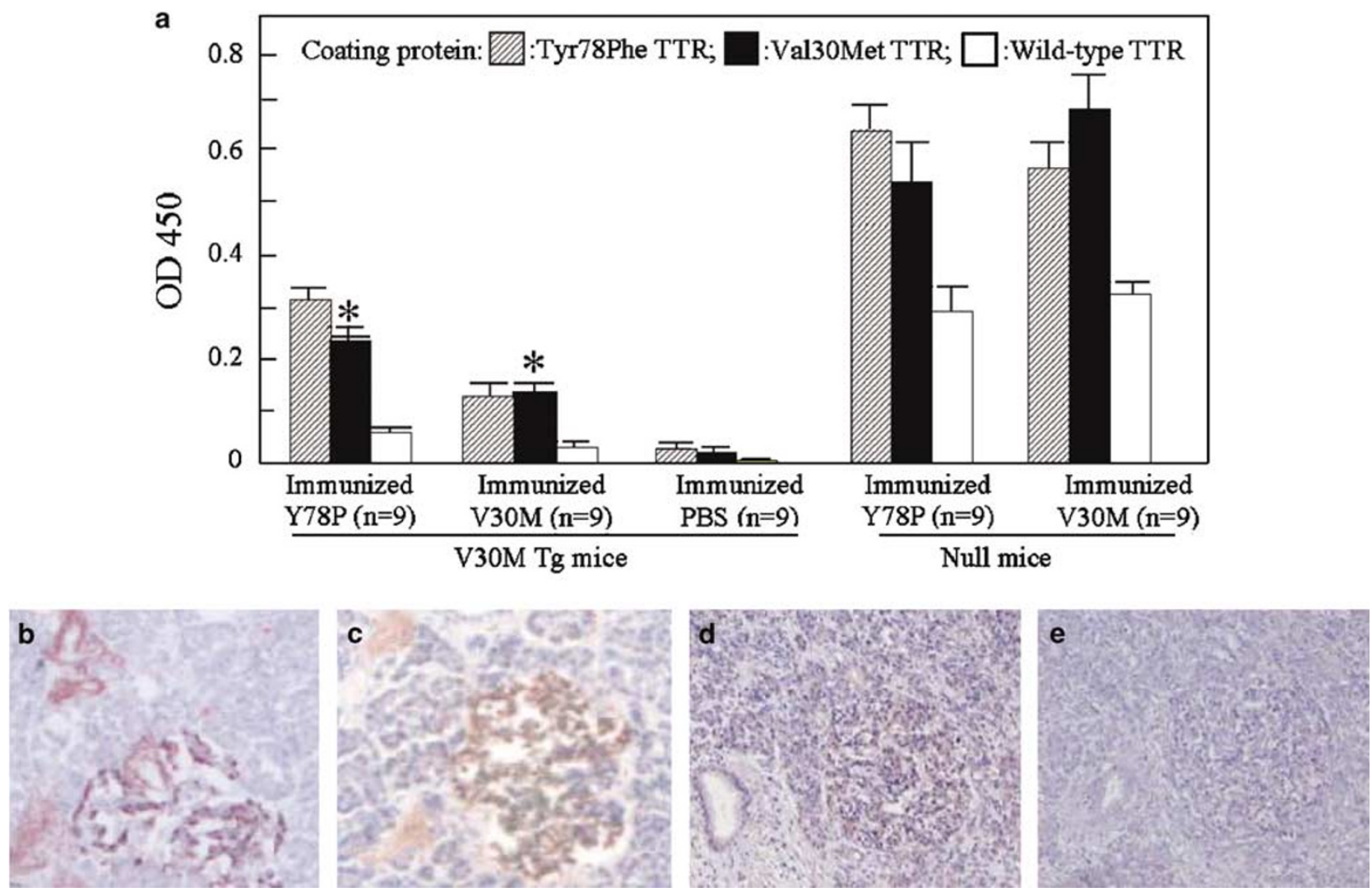

Figure 1 Antibody titers after immunization. (a) Tg mice with (Tg mice) or without (null mice) the V30M TTR gene were immunized with Y78F TTR, V30M TTR, or PBS at 6 months after birth. After 4 months, antibody reactivities against Y78F (hatched columns), V30M (solid columns), and wild-type TTR (white columns) were measured by ELISA, as described in the text. Nine Tg mice with or without the V30M TTR gene were used. (b) Tissue amyloid plaque immunoreactivity. Purified Ig G from immunized Tg mice was tested for reactivity in human pancreas paraffin sections from an FAP patient at 52 years of age who died without liver transplantation (b); (c) shows an adjacent section stained with rabbit anti-human TTR from DAKO; control individual who died from heart disease without pancreas involvement treated with Ig G from immunized Tg mice (d). On parallel sections, Ig G preabsorbed with excess TTR was investigated (e). Magnification: $\times 100$. ${ }^{*}$ Different from wild-type TTR coating.

nerve), no deposition in the form of amyloid was detected. Immunization with V30M did not affect TTR deposition: TTR immunoreactivity was observed in the GI tract (esophagus, stomach, and intestine) in $100 \%$ of the animals (5/5) which did not differ from PBS-immunized mice. In contrast, in the Y78F-immunized mice, scarce TTR deposition was observed in $44 \%$ of the animals (4/9) and the intensity of staining with anti-TTR antibody was much fainter (Figure 2). There was no correlation between the effect of immunization and antibody titers, that is, the degree of TTR removal between animals of each immunized group did not correlate with their antibody titers. This is not unusual, since antibody specificity can prevail over titer.

Quantitative assessment of TTR deposits in the esophagus, stomach, and intestine showed significantly lower values for mice in theY78F TTR immunization group than values found for PBSimmunized mice and mice immunized with V30M TTR (Figure 3a). Therefore, we used Y78F TTR immunization in subsequent experiments at older ages.
To investigate whether $\mathrm{Y} 78 \mathrm{~F}$ immunization prevented and/or affected fibrillar amyloid deposition, tissues from transgenic mice, which started immunization at 12 and 18 months after birth ( $n=9$ in both instances), were analyzed by CR and anti-TTR staining. No amyloid was observed in the Y78F-treated mice both at 16 and 22 months after birth (4 months after immunization), whereas in untreated mice of the same age amyloid deposition was present in approximately $20 \%$ of the animals, particularly in stomach. Each group of Tg mice invariably developed nonfibrillar TTR deposition in extracellular regions in the esophagus, stomach, and intestine. However, according to quantitative analyses, the amounts of TTR deposits were significantly reduced in both the 16 and 22 after birth (4 months after the immunization with Y78F) mice compared with amounts in the PBS-immunized mice (Figure $3 \mathrm{~b}$ ). The reduction in deposition was similar to that observed with immunization at 6 months (Figure 3a). 
Esophagus
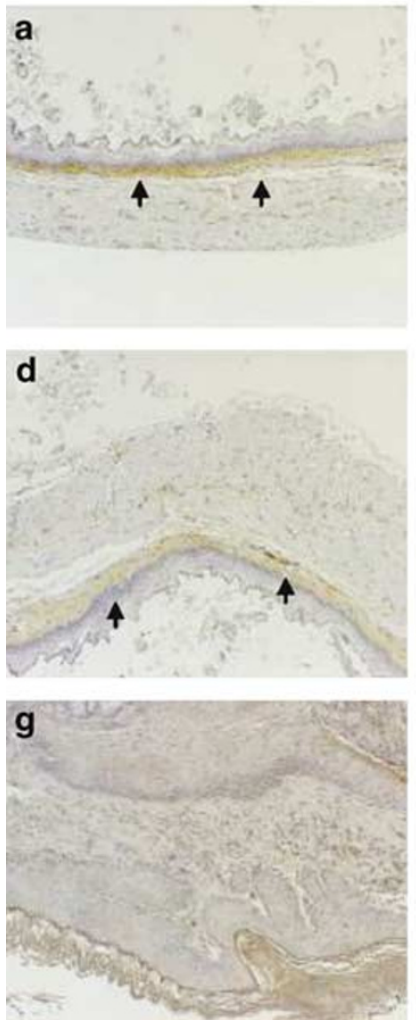

Stomach
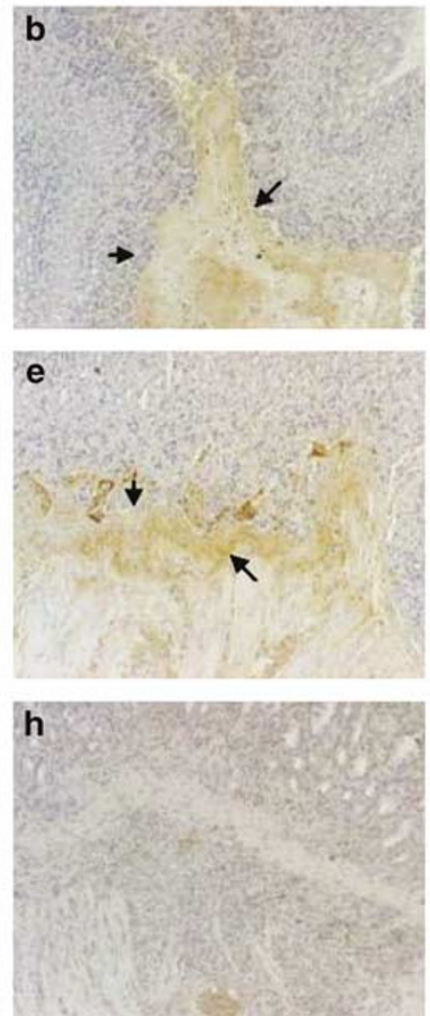

Intestine

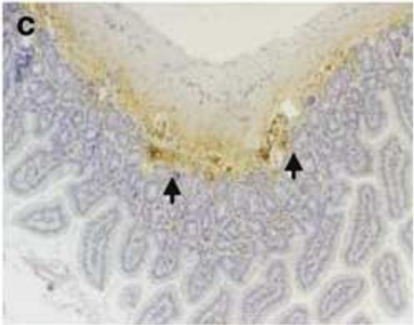

PBS-immunized mice
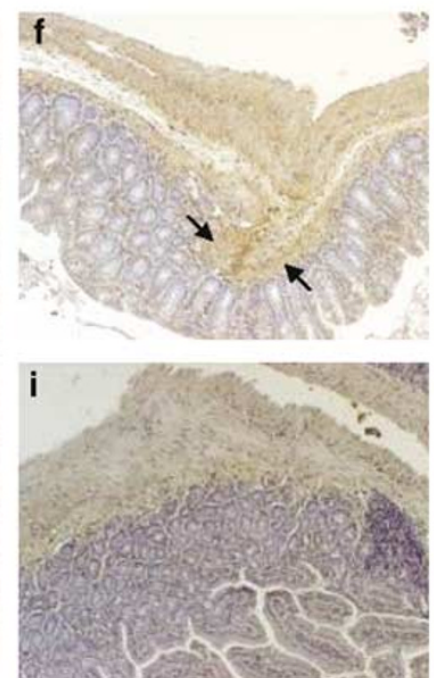

Figure 2 Histopathological changes after immunization. TTR deposits in the esophagus, stomach, and intestine in Tg mice immunized with TTR Y78F, TTR V30M, or PBS. Mice were immunized at 6 months after birth and tissues examined 4 months later. (a-c), TTR deposits in the esophagus, stomach, and intestine of mice injected with PBS. (d-f), TTR deposits in the esophagus, stomach, and intestine of mice immunized with TTRV30M. (g-i), TTR deposits in the esophagus, stomach, and intestine of mice immunized with TTR Y78F. Original magnification for all panels: $\times 200$.

\section{Immunological Responses after Immunization}

In addition to the above-mentioned histopathological changes, tissue specimens from Tg mice immunized with Y78F (in particular, intestines and to a lesser extent stomach) demonstrated inflammatory cell infiltration and hyperplasia of lymphoid tissues only in or near the areas of usual TTR deposition (Figure 4a) which were absent in other areas of the tissue. The extent of infiltration increased with age affecting about $50 \%$ of $\mathrm{Tg}$ mice at 10 months after birth (4 months after the immunization), 64\% of $\mathrm{Tg}$ mice at 16 months after birth (4 months after the immunization), and $89 \%$ of $\mathrm{Tg}$ mice at 22 months after birth (4 months after the immunization). In contrast, mice immunized with M30V had little or no infiltration (data not shown) resembling PBSimmunized mice (Figure $4 \mathrm{~b}$ ) or nontransgenic mice immunized with Y78F (Figure 4c).

To confirm which types of inflammatory cells were present, we performed immunohistochemistry with antibodies to CD5, CD45, and Mac-1, which are surface markers of T cells, B cells, and macrophages, respectively. Most cells in the lymphoid tissues immunereacted predominantly with anti-CD45 antibody but not with anti-CD5 antibody (Figure 4).
Inflammatory cell-infiltrated lesions displayed reaction for anti-Mac-1 antibody (Figure 4), indicating tissue infiltration by macrophages.

\section{IgG Staining in Immunized and NonImmunized Mice}

Immunohistochemistry of stomach, intestine, and esophagus from immunized mice with anti-mouse Ig $G$ antibodies disclosed that these areas of usual TTR deposition contained IgG which was absent in PBSimmunized mice (Figure 5). That is, cells with strong staining for anti-mouse Ig $\mathrm{G}$ antibody were found in sections in which anti-Mac-1 antibody staining also appeared (data not shown). In other areas such as the choroid plexus of brain and Langerhans islets of pancreas, which produce TTR, no inflammatory cells were observed (Figure 5).

\section{Discussion}

In this report, we demonstrated that immunization with TTR Y78F was powerful in reducing TTR deposition and clearing amyloid deposition in an FAP rodent model transgenic for the human mutant V30M with deposition in the gastrointestinal tract. ${ }^{20}$ 

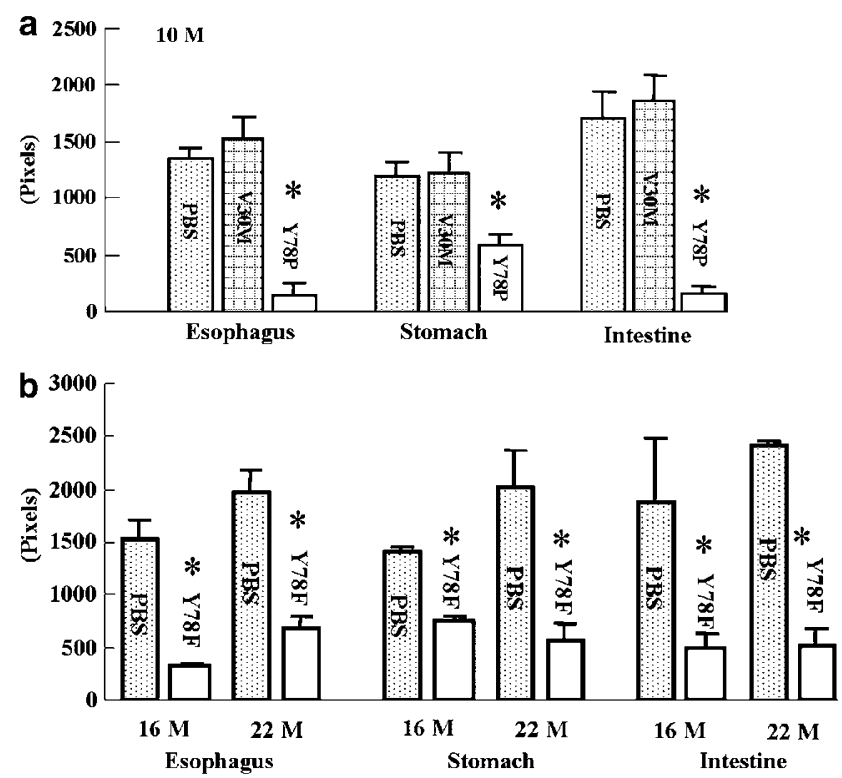

Figure 3 Quantitative evaluation of TTR deposits in immunized and nonimmunized mice. After immunization, the esophagus, stomach, and intestine of mice were examined by histopathological methods, and the densities of immunoreactivities in these organs after reaction with anti-human TTR antibody were determined by semiquantitative image analysis. Values for each group are means \pm s.d. Asterisks indicates statistical significance. ${ }^{*} P<0.02$. (a) Mice at 10 months after birth (4 months after the immunization with the indicated antigens or PBS). (b) Tg mice at 16-, and 22 months after birth (4 months after the immunization) immunized with PBS alone or Y78F.

In general, the mammalian immune system does not generate antibodies against intrinsic peptides and proteins because of the immune tolerance system, provided that structural alterations do not occur. Studies by Monsonego et al demonstrated that administration of synthetic fragments of $\mathrm{A} \beta_{1-15}$ conjugated to bovine serum albumin induced highly significant antibody production in a murine Alzheimer's model, but the amyloid $\beta(\mathrm{A} \beta)$ fragment alone did not produce a significant amount of antibody. ${ }^{24}$ Conjugation of the $\mathrm{A} \beta$ fragment to bovine serum albumin or some carrier protein may be required to overcome the immune tolerance system in vivo. In our experiments, immunization with TTR V30M, an intrinsic protein found in FAP patients, could not induce a high antibody titer in Tg mice possessing the human V30M TTR gene and did not prevent TTR and amyloid deposition in these mice. By contrast, immunization of Tg mice with TTR Y78F produced a higher titer of antibodies against Y78F TTR in serum, than with V30M TTR, suggesting significant structural changes of the Y78F mutant comparative to the V30M mutant, although some epitopes might be shared as suggested by the positive reaction of isolated Y78F antibodies to TTR-synthesizing tissues, such as the pancreas.

The immunization protocol was effective in all tested ages, that is, resulted in a drastic reduction in the extent of TTR deposition as nonfibrillar materi- al, before overt amyloid formation and cleared amyloid, because congophilic material was absent upon treatment of older animals that normally present with amyloid. Thus, it could potentially be applied in FAP at early stages, at least to clear deposition in peripheral non-neuronal tissues; we have shown nonfibrillar TTR deposition in tissues of asymptomatic carriers of the V30M mutation before overt amyloid deposition was diagnosed as analyzed by CR and electron microscopy. ${ }^{25} \mathrm{At}$ the moment, all attempts to generate animal FAP rodent models with deposition in the nerve have failed and the current model has predominance for GI deposition. At any rate, the available models are very valuable to test approaches to counteract nonfibrillar and fibrillar TTR deposition.

Different results suggest that the generated antibodies in each Tg mice immunized with Y78F are basically polyclonal and contain different IgGs reacting with different nonconformational epitopes of TTR; thus, by sandwich ELISA, the antibodies from Y78F-immunized mice recognized WT and V30M and when tested by Western blot they react with recombinant and plasma TTR from V30M carriers and noncarriers equally (not shown); furthermore, as shown in Figure 1d, they recognize soluble human TTR synthesized by human pancreas pointing out for nonconformational epitopes. However, some of them bind primarily TTR deposits in tissues as shown by the presence of anti-mouse IgG in tissues with TTR deposition, possibly recognizing conformational epitopes. Also, the abundance of B cells in tissue sections and the paucity of $\mathrm{T}$ cells could suggest that these are T-independent antigens.

At this point, it is difficult to clearly discern the molecular mechanisms underlying the effects observed that can preclude both prevention and clearance of deposition. However, the infiltration of a large number of $B$ cells and macrophages and the detection of IgG in lesions where TTR and amyloid deposits normally appear suggest a clearance mechanism involving FcR-mediated phagocytosis, as earlier reported in the Alzheimer immunization protocols. ${ }^{15}$ However, a recent study indicated that FcR-mediated uptake of anti-A $\beta$ immune complexes is not required for the attenuation of $\mathrm{A} \beta$ deposition after $\mathrm{A} \beta 1-42$ immunization in Tg mice. ${ }^{26}$ McLaurin et $a 1^{27}$ demonstrated antibody inhibition of $\mathrm{A} \beta$ fibrillogenesis and cytotoxicity without eliciting an inflammatory response. We speculate that not only FcR-mediated phagocytosis through Ig $\mathrm{G}$ but also non-FcR-mediated clearance mechanism for amyloid deposition might as well effectively attenuate TTR deposition in V30M Tg mice.

On the basis of the experiments with the murine Alzheimer disease model, immunization therapy with $\mathrm{A} \beta$ peptide has been started in patients with Alzheimer's disease who are in the early to moderate stage of the disease. Unfortunately, the first clinical trial using $\mathrm{A} \beta_{1-42}$ peptide (AN 1792 produced by ELAN Pharmaceuticals) with adjuvant QS-21 for 

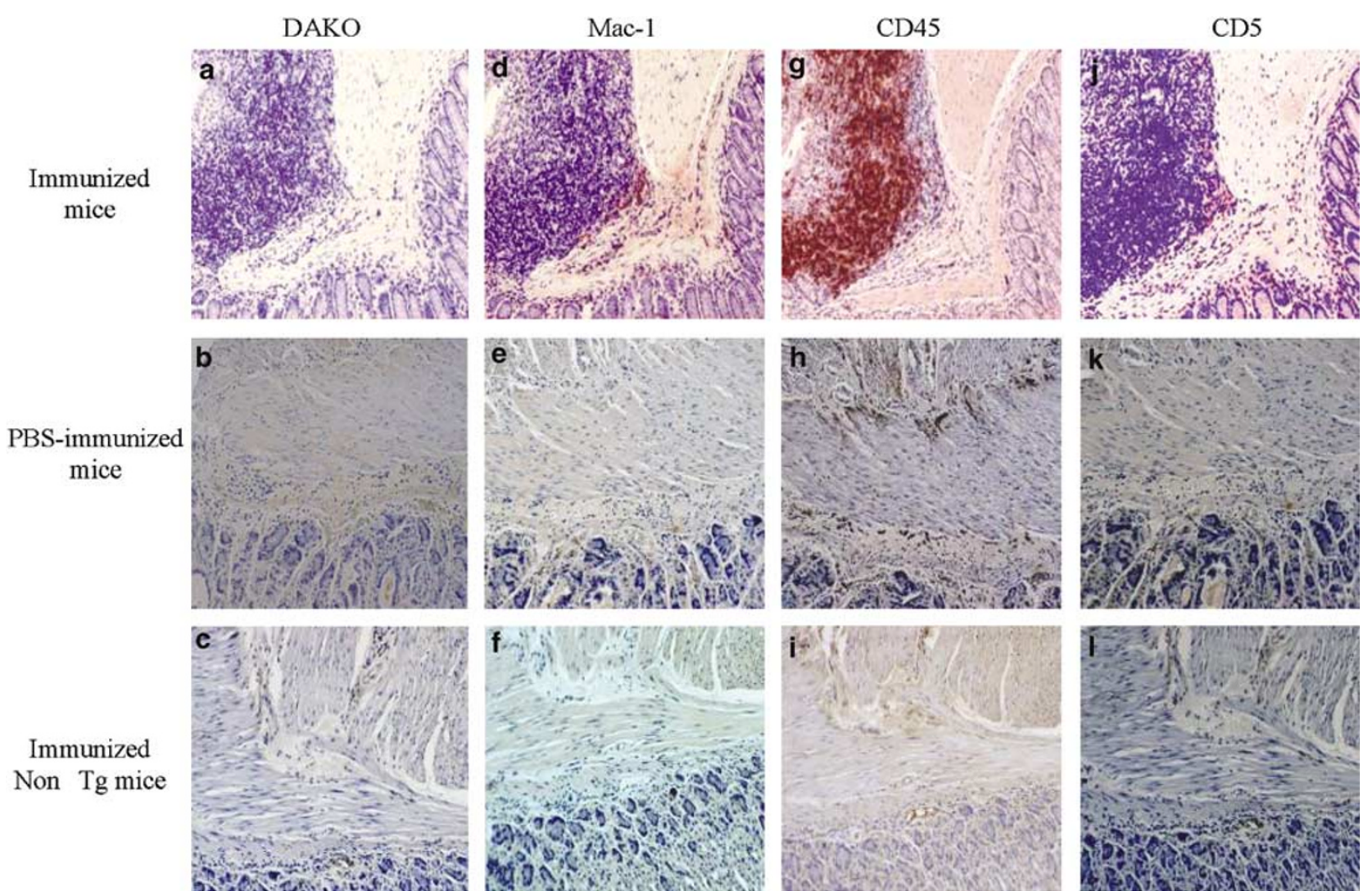

Figure 4 Immunological responses after immunization. (a-c) Immunoreactivity with polyclonal anti-human TTR antibody of intestine of Tg mice carrying the V30M gene immunized with Y78F TTR (immunized mice) at 16 months after birth (4 months after the immunization) or with PBS (PBS-immunized mice), and TTR-null mice at 16 months after birth (4 months after the immunization) immunized withY78F TTR (immunized non-Tg mice), respectively. Immunoreactivities for different antigens in adjacent sections: (d-f) with rat monoclonal anti-mouse Mac-1 antibody; (g-i) with rat monoclonal anti-mouse CD45 antibody; (j,-l) with rat monoclonal antimouse CD5 antibody. Original magnification for all panels $\times 200$.

\section{Non-Immunized \\ Tg mice}
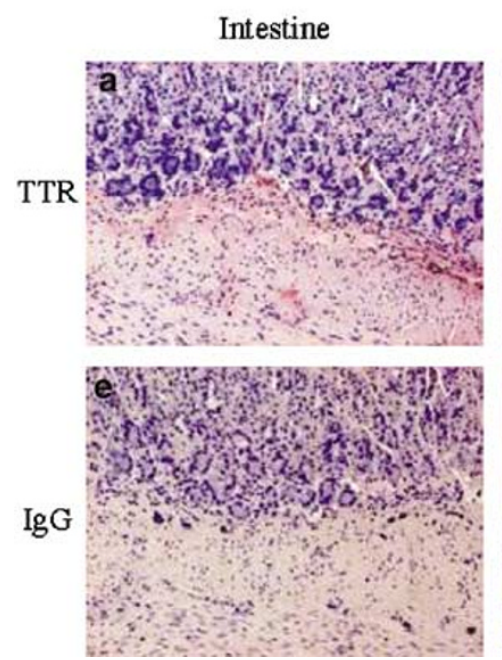

\section{Immunized Tg mice}
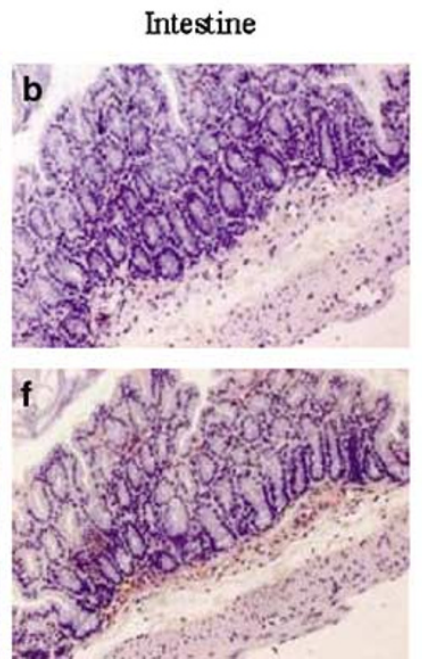

Pancreas
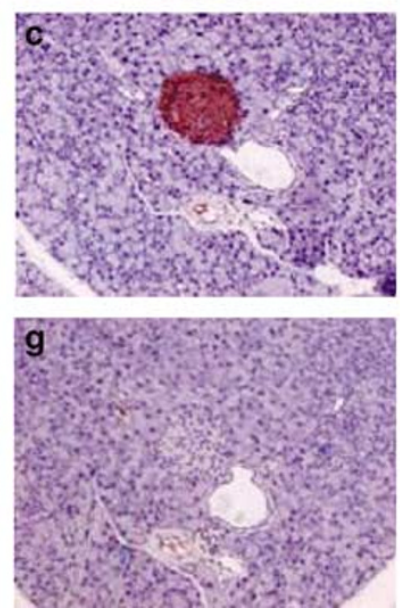

Choroid plexus
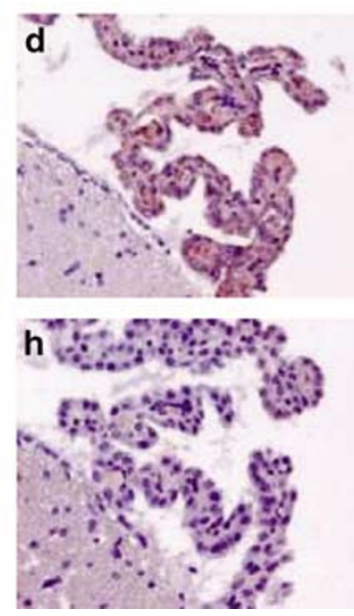

Figure 5 IgG staining in immunized and PBS-immunized mice. (a-d) Immunoreactivity with polyclonal anti-human TTR antibody in the intestine of Tg mice carrying the V30M TTR gene injected with PBS at 16 months after birth (4 months after the immunization) and in the intestine, pancreas, and choroid plexus of the same strain immunized with Y78F TTR at 16 months after birth (4 months after the immunization), respectively. (e-h) Immunoreactivity with anti-mouse IgG antibody in adjacent sections . Original magnification for all panels $\times 100$. 
375 patients was suspended because spinal tap results from 17 patients showed elevated protein and lymphocyte levels that seemed to be associated with a nonviral allergic reaction. ${ }^{28}$ It is speculated that this phenomenon was caused by a direct immunoallergic response against the amyloid precursor protein (APP), which is expressed in the brain and peripheral tissues or to A $\beta .^{29}$ As APP was amyloidogenic, and intrinsic proteins may also be so, better methodologies or modified antigens for immunization are required to treat amyloid deposition. We found no evidence for antibodies in TTR V30M-producing cells, such as those of the choroid plexus and Langerhans islets and no inflammatory cell infiltration was observed in and around these areas, suggesting that our strategy might pose no problems with immunoallergic responses. In this regard, passive immunization using a humanized antibody recognizing specifically amyloidogenic TTR should be attempted.

In conclusion, we found that immunization with Y78F TTR prevented/cleared nonfibrillar and fibrillar TTR deposition by activation of phagocytes. In addition, we observed no pathological changes in the tissues, except for lesions related to TTR and amyloid deposits. Thus, this therapy seemed to have no harmful physiological effect on mice and may become a useful novel therapeutic tool for FAP in the near future. The target for this therapy may be applied in FAP patients with significant amyloid deposition in tissues. In addition, the therapy may be used as a vaccination for healthy TTR variant gene carriers. As for recipients of a domino FAP liver, this immunization therapy may not be effective because these individuals receive immunosuppressants.

\section{Acknowledgements}

We thank Rossana Correia and Paul Moreira for excellent technical support in performing tissue processing and protein isolation, respectively. $\mathrm{H}$ Terazaki was a recipient of a fellowship from Fundação Oriente (Portugal) and from the Ministry of Education, Science, Sports and Culture of Japan. This work was funded by grants from Fundação Ciencia e Tecnologia (FCT) and Gulbenkian Foundation, Portugal.

\section{References}

1 Dobson CM. Protein folding and its links with human disease. Biochem Soc Sym 2001;68:1-26.

2 Selkoe DJ. Amyloid beta-protein and the genetics of Alzheimer's disease. J Biol Chem 1996;271:1829518298.

3 Weissmann C, Enari M, Klohn PC, et al. Transmission of prions. J Infect Dis 2002;186:157-165.

4 Saraiva MJ. Transthyretin amyloidosis: a tale of weak interactions. FEBS Lett 2002;498:201-203.
5 Andrade C. A peculiar form of peripheral neuropathy; familiar atypical generalized amyloidosis with special involvement of the peripheral nerves. Brain 1952; $75: 408-427$.

6 Ando Y, Araki S, Ando M. Transthyretin and familial amyloidotic polyneuropathy. Intern Med 1993;32: 920-922.

7 de Carvalho M, Conceição I, Bentes C, et al. Long-term quantitative evaluation of liver transplantation in familial amyloid polyneuropathy (Portuguese V30M). Amyloid 2002;9:126-133.

8 Suhr OB, Ericzon BG, Friman S. Long-term follow-up of survival of liver transplant recipients with familial amyloid polyneuropathy (Portuguese type). Liver Transpl 2002;8:787-794.

9 Haraoka K, Ando Y, Ando E, et al. Presence of variant transthyretin in aqueous humor of a patient with familial amyloidotic polyneuropathy after liver transplantation. Amyloid 2002;9:247-251.

10 Ando E, Ando Y, Haraoka K. Ocular amyloid involvement after liver transplantation for polyneuropathy. Ann Intern Med 2001;135:931-932.

11 Altland K, Winter P, Saraiva MJ, et al. Sulfite and base for the treatment of familial amyloidotic polyneuropathy: two additive approaches to stabilize the conformation of human amyloidogenic transthyretin. Neurogenetics 2004;8:61-67.

12 Oza VB, Smith C, Raman P, et al. Synthesis, structure, and activity of diclofenac analogues as transthyretin amyloid fibril formation inhibitors. J Med Chem 2002;45:321-332.

13 Cardoso I, Merlini G, Saraiva MJ. 4'-Iodo-4'-deoxydoxorubicin and tetracyclines disrupt transthyretin amyloid fibrils in vitro producing noncytotoxic species: screening for TTR fibril disrupters. FASEB J 2003; 17:803-809.

14 Schenk D, Barbour R, Dunn W, et al. Immunization with amyloid- $\beta$ attenuates Alzheimer-disease-like pathology in the PDAPP mouse. Nature 1999;400: 173-177.

15 Bard F, Cannon C, Barbour R, et al. Peripherally administered antibodies against amyloid $\beta$-peptide enter the central nervous system and reduce pathology in a mouse model of Alzheimer disease. Nat Med 2000;6:916-919.

16 Sigurdsson EM, Brown DR, Daniels M, et al. Immunization delays the onset of prion disease in mice. Am J Pathol 2002;161:13-17.

17 Goldsteins G, Persson H, Andersson K, et al. Exposure of cryptic epitopes on transthyretin only in amyloid and in amyloidogenic mutants. Proc Natl Acad Sci USA 1999;96:3108-3113.

18 Redondo C, Damas AM, Olofsson A, et al. Search for intermediate structures in transthyretin fibrillogenesis: soluble tetrameric Tyr78Phe TTR expresses a specific epitope present only in amyloid fibrils. J Mol Biol 2000;304:461-470.

19 Hock C, Konietzko U, Papassotiropoulos A, et al. Generation of antibodies specific for $\beta$-amyloid by vaccination of patients with Alzheimer disease. Nat Med 2002;8:1270-1275.

20 Kohno K, Palha JA, Miyakawa K, et al. Analysis of amyloid deposition in a transgenic mouse model of homozygous familial amyloidotic polyneuropathy. Am J Pathol 1997;150:1497-1508.

21 Sousa MM, Fernandes R, Palha JA, et al. Evidence for early cytotoxic aggregates in transgenic mice for 
human transthyretin Leu55Pro. Am J Pathol 2002; 161:1935-1948.

22 Episkopou V, Maeda S, Nishiguchi S, et al. Disruption of the transthyretin gene results in mice with depressed levels of plasma retinol and thyroid hormone. Proc Natl Acad Sci USA 1993;90:2375-2379.

23 Puchtler H, Sweat F. Congo red as a stain for fluorescence microscopy of amyloid. J Histochem Cytochem 1965;13:693-694.

24 Monsonego A, Maron R, Zota $\mathrm{V}$, et al. Immune hyporesponsiveness to amyloid $\beta$-peptide in amyloid precursor protein transgenic mice: implications for the pathogenesis and treatment of Alzheimer's disease. Proc Nat Acad Sci USA 2001;98:10273-10278.

25 Sousa MM, Cardoso I, Fernandes R, et al. Deposition of transthyretin in early stages of familial amyloidotic polyneuropathy: evidence for toxicity of nonfibrillar aggregates. Am J Pathol 2001;159:1993-2000.

26 Das $\mathrm{P}$, Howard $\mathrm{V}$, Loosbrock $\mathrm{N}$, et al. Amyloid- $\beta$ immunization effectively reduced amyloid deposition in FcR $\gamma-/$ - knock-out mice. J Neurosci 2003;23:85328538.

27 McLaurin J, Cecal R, Kierstead ME, et al. Therapeutically effective antibodies against amyloid- $\beta$ peptide target amyloid- $\beta$ residues 4-10 and inhibit cytotoxicity and fibrillogenesis. Nat Med 2002;8:1263-1269.

28 Schenk D. Amyloid $\beta$ immunotherapy for Alzheimer's disease: the end of the beginning. Nat Rev Neurosci 2002;3:824-828.

29 Senior K. Dosing in phase II trial of Alzheimer's vaccine suspended. Lancet Neurol 2002;1:3. 